\title{
Subwavelength Grating Devices in SOI
}

\author{
Junjia Wang ${ }^{1}$, Reza Ashrafi ${ }^{1}$, Ivan Glesk ${ }^{2}$, and Lawrence R. Chen ${ }^{1}$ \\ ${ }^{1}$ Department of Electrical and Computer Engineering, McGill University, Montreal, QC H3A OE9 Canada \\ ${ }^{2}$ Department of Electronic and Electrical Engineering, University of Strathclyde, Glasgow, G1 1XU, UK
}

\begin{abstract}
We demonstrate subwavelength grating devices in silicon-on-insulator including Bragg gratings, racetrack resonators, and optical delay lines. The Bragg grating filter has a 3 $\mathrm{dB}$ bandwidth of $0.5 \mathrm{~nm}$ and a reflectivity of $90 \%$; the racetrack resonator has a $3 \mathrm{~dB}$ bandwidth of $1 \mathrm{~nm}$, a free spectral range of $4.6 \mathrm{~nm}$, and an extinction ratio as high as $33 \mathrm{~dB}$; the optical delay lines achieve $~ 30$ ps time-delay with $30 \%$ change in duty-cycle.
\end{abstract}

\section{Introduction}

There is an increasing need for integrated solutions in optical communications and interconnections applications [1]. In the past few years, a variety of active and passive devices as well as integrated subsystems in CMOS-compatible silicon photonics platforms have been realized [2]. Subwavelength grating (SWG) waveguides have attracted interest due to their potential for low loss and flexibility in tailoring the effective index $[3,4]$. Indeed, a number of SWG-based devices/building blocks in silicon-on-insulator (SOI) have been developed, including waveguide crossings [3], bends [4], couplers [4-6], and ring resonators [7]. Bragg gratings (BGs) and ring resonators have important applications as optical filters and for implementation in more complex wavelength selective devices. Optical delay line (ODL) devices are fundamental building blocks in all-optical and microwave photonic signal processing circuits. In this paper, we demonstrate SWG BG filters, racetrack resonators, and ODLs.

\section{SWG BGs}

Fig. 1a compares the schematic of an SWG waveguide and an SWG BG in SOI. The effective index of the SWG waveguide depends on the duty cycle $f=a / \Lambda$, where $a$ is the width of the high index medium (here $\mathrm{Si}$ ) and $\Lambda$ is the period. An SWG BG can then be realized by interleaving two SWG waveguides with different duty cycles $f_{1}=$ $a_{1} / \Lambda_{1}$ and $f_{2}=a_{2} / \Lambda_{2}$; the SWG BG has a period of $\Lambda_{1}+\Lambda_{2}$. By varying $f_{l}$ and $f_{2}$, we create a periodic variation in the effective index and can thus obtain Bragg reflection. The cross-section of the SWG waveguides used in our experiments is shown in Fig. $1 b$. The width of the silicon layer is $500 \mathrm{~nm}$ and the thickness is $220 \mathrm{~nm}$; it sits on top of a $3 \mu \mathrm{m}$ thick buried oxide (BOX) layer and is covered by an index-matched $2 \mu \mathrm{m}$ thick oxide cladding. We consider SWG waveguides with $\Lambda=280 \mathrm{~nm}$ to obtain a transmission window spanning the C-band and 1,000 periods. Two SWG tapers are used to convert light into (and from) a Bloch mode before (and after) propagating through the SWG waveguide [5]. Vertical grating couplers (VGCs) [8] optimized for TE transmission are used to couple light in and out of the device. A compact Y-branch is used to extract the reflection response of the SWG BGs. The devices were fabricated using electron beam lithography with a single full etch. Figs. $1 c$ and $1 d$ show the SEM image of an SWG BG (before deposition of the top oxide cladding) and the full layout of the device.

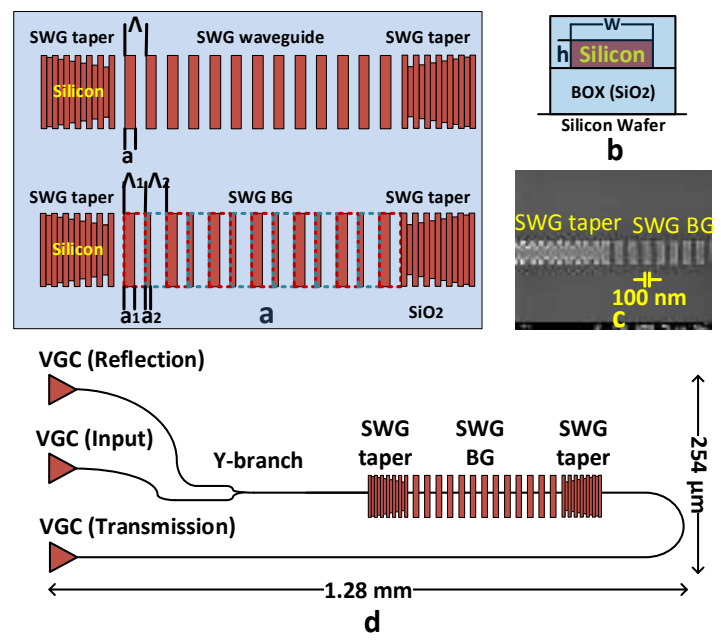

Fig. 1. (a) Top view of an SWG waveguide and an SWG BG, (b) device cross-section, (c) SEM of the fabricated SWG BG prior to top oxide cladding deposition, and (d) full layout.

Fig. $2 a$ shows the measured transmission response of the SWG waveguide without and with an index modulation based on $f_{1}=50 \%$ and $f_{2}=$ $48 \%$. There are no spectral features within the transmission window of the SWG waveguide; on the other hand, the SWG BG exhibits a clear rejection peak at a resonant wavelength of 1546.8 $\mathrm{nm}$. Fig. $2 b$ shows a zoom of the transmission and reflection responses around $1546.8 \mathrm{~nm}$ : the transmission loss is $-12 \mathrm{~dB}$ corresponding to a peak reflectivity of $90.4 \%$; the $3 \mathrm{~dB}$ bandwidth is $0.5 \mathrm{~nm}$. 
The total fiber-to-fiber loss is typically $15 \mathrm{~dB}$ (input port to reflection port).
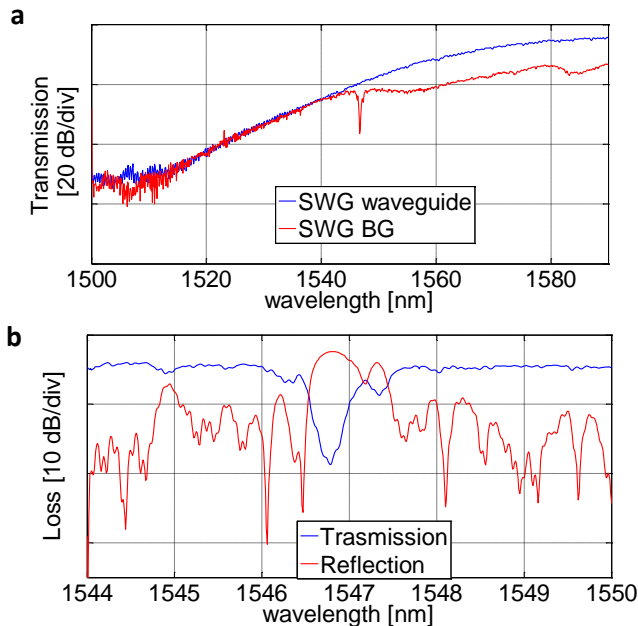

Fig. 2. Measured response of SWG waveguide and SWG BG $\left(f_{I}\right.$ $=50 \%$ and $f_{2}=48 \%$ ).

\section{SWG Racetrack Resonators}

In [5], we demonstrated SWG ring resonators that are under coupled. Racetrack resonators are preferable since the coupling region between the resonator and bus waveguides is longer compared to ring configurations, thereby allowing for greater control over coupling efficiency. Figs. $3 a$ and $3 b$ show the device layout and a zoom of the SWG racetrack resonator. The length of the directional coupling section is $L$ and the separation between the bus and racetrack waveguides (center-to-center) is denoted $g$; these two parameters determine the coupling efficiency and operating condition (under coupled, critically coupled, over coupled) of the resonator. The curved section has a radius $r$ and the total perimeter of the racetrack which determines the free spectral range (FSR) is $2 \pi r+2 L$. We use the same waveguide cross-section and VGC designs as for the SWG BGs. The SWG period is $300 \mathrm{~nm}$ and the duty cycle is $50 \%$ (to obtain a transmission window in the $\mathrm{C}$-band).

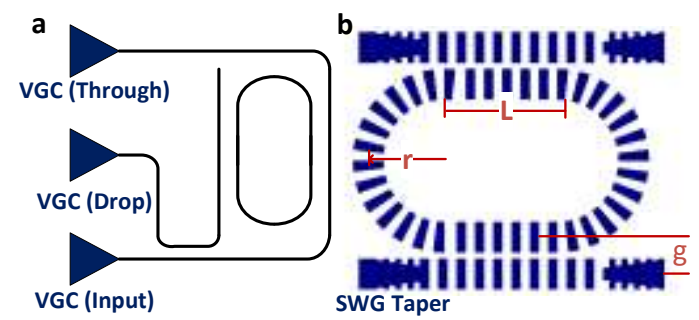

Fig. 3. Schematic of the (a) device layout, and (b) SWG racetrack resonator.

Fig. 4 shows the measured through and drop responses of an SWG racetrack resonator with $r=$
$20 \mu \mathrm{m}, L=40 \mu \mathrm{m}$, and $g=600 \mathrm{~nm}$. The measured FSR is $4.6 \mathrm{~nm}$, which agrees well with the value calculated using the total perimeter of the racetrack and effective waveguide index of 2.47. At 1527.9 $\mathrm{nm}$, near critical coupling is achieved and we obtain an extinction ratio (ER) as high as $33 \mathrm{~dB}$; the $3 \mathrm{~dB}$ bandwidth is $1 \mathrm{~nm}$. The amplitude and ER variations are due to the wavelength dependence of the directional coupling.
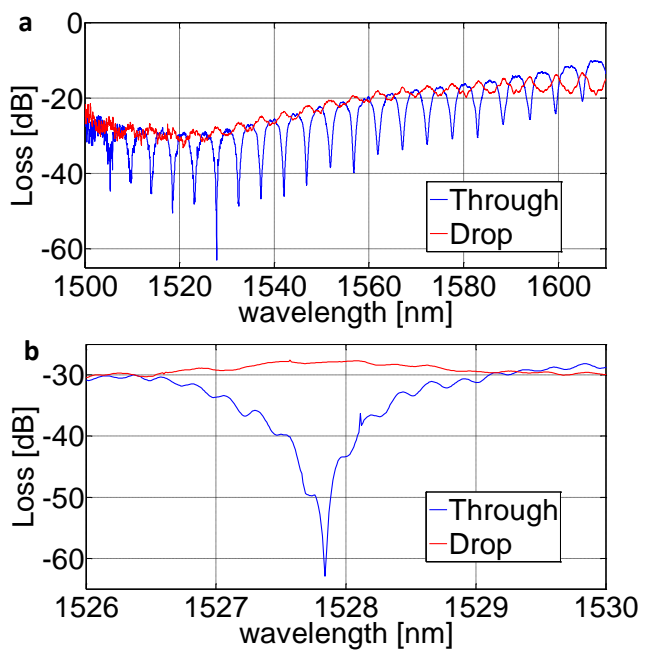

Fig. 4. (a) Measured through and drop responses of a SWG racetrack resonator with $r=20 \mu \mathrm{m}, \mathrm{L}=40 \mu \mathrm{m}$, and $g=600 \mathrm{~nm}$. (b) Zoom in at one resonance at $1527.85 \mathrm{~nm}$.

\section{SWG Delay Lines}

By choosing the duty cycle $f$, the effective (and group) index of the SWG waveguide can be modified and hence, optical delay can be realized. Fig. 5a shows a schematic of our proof-of-principle demonstration, which is a 4-tap ODL device on silicon-on-insulator (SOI) platform. The effective refractive index of the propagating Bloch mode in each tap of the fabricated ODL is a function of $f$. See [7] for a detailed numerical analysis about this. The corresponding generated time-delay difference between the taps of the ODL device can be expressed as

$$
\Delta t(i)=\left[n_{e}(i+1)-n_{e}(i)\right] \cdot L / c, \quad 1<i<N-1
$$

where $c$ is the light speed in vacuum, and $N=4$ is the number of taps in the ODL. By properly designing the duty cycle $f_{i}$ in each waveguide, the temporal separation between the taps can be controlled. Fig. 5b shows an SEM image of our fabricated device. The waveguide cross-section and VGC designs are the same as for the SWG BGs. The SWG period is $250 \mathrm{~nm}$ (to reduce losses and obtain a transmission window in the C-band). The designed duty cycles in SWG waveguides are $f_{1}=60 \%, f_{2}=50 \%, f_{3}=40 \%$, and $f_{4}=30 \%$. Six Ybranches (with 50:50 splitting ratio) were used to 
split and collect the optical power at the input and output of the SWG waveguides, respectively.
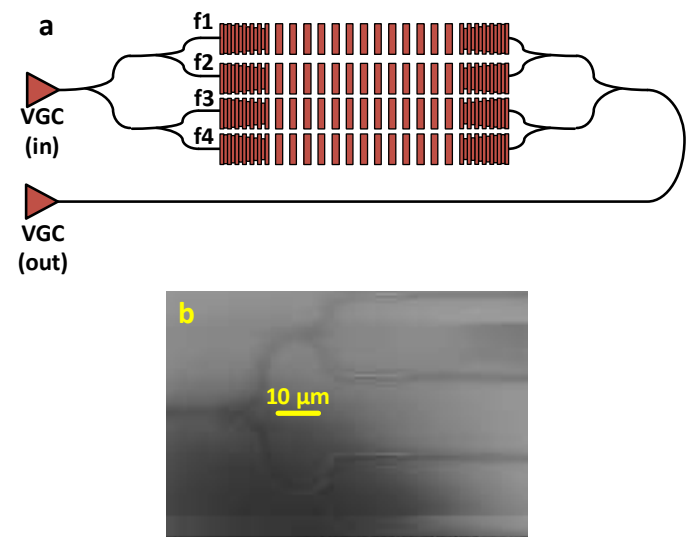

Fig. 5. (a) Schematic and (b) an SEM image of our fabricated 4tap ODL structure based on SWG waveguides in SOI.

Fig. 6 shows the measured spectral response of the fabricated ODL device. At the input, we lunch a Gaussian-like optical pulse with a full width at half maximum (FWHM) bandwidth of $2 \mathrm{~nm}$ at a central wavelength of $1556 \mathrm{~nm}$, which is generated using an actively mode-locked fiber laser with a repetition rate of $10 \mathrm{GHz}$. Fig. 7 shows the generated timedomain output pulse train, measured using optical sampling oscilloscope. The signal spectrum at the input and output of the ODL device is also shown in the inset of Fig. 7. As it can be seen in Fig. 7, the generated time-delay difference between the taps of the ODL by introducing $10 \%$ change in duty cycles of SWG waveguides with $8 \mathrm{~mm}$ length is $\sim 9 \mathrm{ps}$. According to Eq. (1), this time-delay difference between the taps corresponds to an effective refractive index difference of 0.338 between the SWG waveguides. Note that as shown in Fig. 3, the pulse propagating through the SWG waveguide with lowest duty cycle (i.e. $D_{4}$ ) arrives faster than the other branches. The radiation loss increases as we decrease the duty cycle [9]. This can be seen in Fig. 7, where the output pulse corresponding to the waveguide branch with $f_{4}=30 \%$ has the most amount of propagation loss. This additional loss can be compensated by properly designing the Ybranches with non 50:50 splitting ratios.

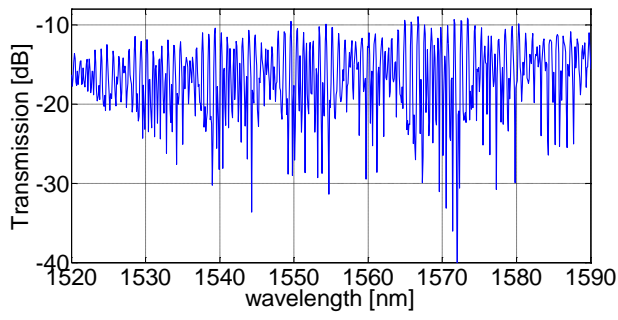

Fig. 6. Measured spectral response of the fabricated ODL.

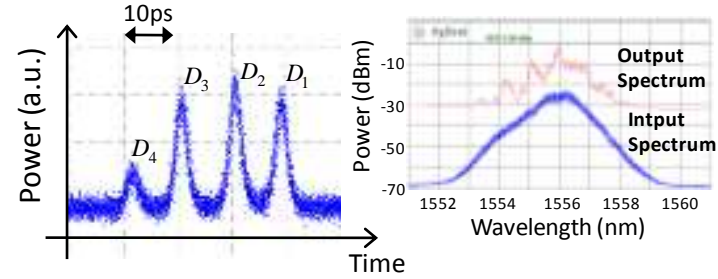

Fig. 7. Generated pulse train at the output of the fabricated ODL device in response to a single input optical pulse. Input and output signal spectra have also shown in the inset.

\section{Conclusions}

We have demonstrated and experimentally verified SWG filters in SOI based on BGs and racetrack resonators. The SWG BG exhibits high reflectivity (> 90\%) and a relatively narrow reflection bandwidth $(0.5 \mathrm{~nm})$. The SWG racetrack resonator exhibits an extinction ratio as high as $33 \mathrm{~dB}$. We also proposed a novel ODL approach based on SWG waveguides in SOI. The obtained results clearly prove a great capability of the SWG waveguides to be employed in ODLs to achieve a considerably compact structure. These devices are compatible with other SWG building blocks and can be used to develop more complex structures with enhanced functionality for applications in communications and sensing.

\section{Acknowledgments}

The devices were fabricated by R. Bojko at the University of Washington Nanofabrication Facility, a member of the NSF National Nantotechnology Infrastructure Network.

\section{References}

1. Y. Arakawa et al, Ieee Commun Mag 51, $72-77$ (2013).

2. Y. A. Vlasov, Ieee Commun Mag 50, S67-S72 (2012).

3. P. J. Bock et al, Optics Express 18, 16146-16155 (2010).

4. V. Donzella et al, Optics Express 22, 21037-21050 (2014).

5. R. Halir et al, Opt. Express 20, 13470-13477 (2012).

6. A. Ortega-Monux et al, Photonics Technology Letters, IEEE 23, 1406-1408 (2011).

7. J. Wang et al, Optics Express 22, 15335-15345 (2014).

8. Y. Wang et al, Optics Express 22, 20652-20662 (2014).

9. D. Ortega et al, J Lightwave Technol 17, 369-375 (1999). 